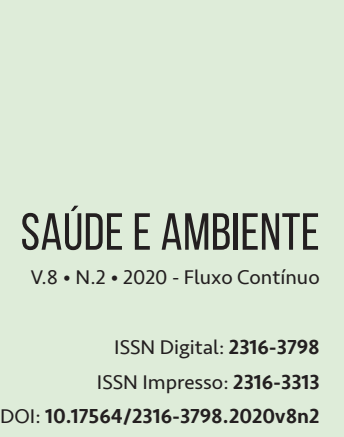

DOI: $10.17564 / 2316-3798.2020 v 8 n 2$

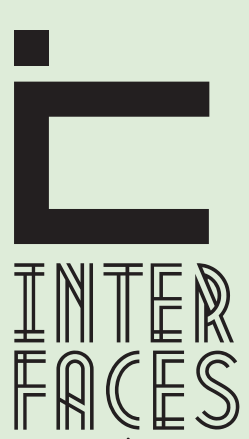

CIENTÍFICAS

\title{
CARACTERIZAÇ̃̃o DO PERFIL CLÍNICO E SOCIODEMOGRÁFICO DE PACIENTES ADMITIDOS EM UMA UNIDADE DE TERAPIA INTENSIVA
}

\author{
CHARACTERIZATION OF THE CLINICAL AND \\ SOCIODEMOGRAPHIC PROFILE OF PATIENTS ADMITTED \\ IN A UNIT OF INTENSIVE THERAPY
}

CARACTERIZACIÓN DEL PERFIL CLÍNICO Y SOCIODEMOGRÁFICO DE PACIENTES INGRESADOS EN UNIDAD DE CUIDADOS INTENSIVOS

Cleidinaldo Ribeiro de Goes Marques ${ }^{1}$ Marcos Rodrigues Santos ${ }^{2}$ Krislainy Santos Passos ${ }^{3}$ Shirley Dósea dos Santos Naziazeno ${ }^{4}$ Lucas Andrade de Sá ${ }^{5}$ Eduesley Santana Santos ${ }^{6}$

\section{RESUMO}

O objetivo deste estudo é caracterizar o perfil clínico e sociodemográfico de pacientes admitidos em uma Unidade de Terapia Intensiva de um hospital do estado de Sergipe. Trata-se de um corte transversal de uma coorte prospectiva vinculada ao projeto intitulado UTISE. A coleta de dados ocorreu diariamente, entre outubro/2018 e maio/2019, sendo realizada por meio da análise dos prontuários eletrônicos. A amostra da pesquisa foi por conveniência e correspondeu aos pacientes admitidos durante o período de coleta de dados. Foram incluídos 43 pacientes, predominantemente do sexo masculino (55,8\%), com uma média de idade de $68 \pm 19,25$ anos, peso de $67,53 \pm 13,28 \mathrm{~kg}$, índice de massa corpórea de $24,66 \pm 6,38 \mathrm{~kg} / \mathrm{m}^{2}$, altura entre 1,60-1,69 m, grande parte dos pacientes procedentes da emergência $66,1 \%$, sendo os distúrbios cardiovasculares $25,1 \%$ o principal motivo de internação. A partir da identificação deste perfil torna-se possível a utilização dos resultados deste estudo como subsídio para novas pesquisas em outras instituições hospitalares, bem como prover um melhor cuidado e mais adequado para o tipo identificado.

\section{PALAVRAS-CHAVE}

Unidades de Terapia Intensiva. Cuidados Críticos. Indicadores de Morbimortalidade. 


\section{ABSTRACT}

The aim of this study is to characterize the clinical and sociodemographic profile of patients admitted to an Intensive Care Unit of a hospital in the state of Sergipe. This is a cross-section of a prospective cohort linked to the project entitled UTISE. Data collection occurred daily, between October / 2018 and May / 2019, and was performed through the analysis of electronic medical records. The research sample was for convenience and corresponded to patients admitted during the data collection period. 43 patients were included, predominantly male (55.8\%), with an average age of $68 \pm 19,25$ years, weight of $67,53 \pm 13,28 \mathrm{~kg}$, body mass index of $24,66 \pm 6,38 \mathrm{~kg} / \mathrm{m}^{2}$, height between $1.60-1.69 \mathrm{~m}$, most patients coming from the emergency room $66.1 \%$, with cardiovascular disorders $25.1 \%$ being the main reason for hospitalization. From the identification of this profile, it is possible to use the results of this study as a subsidy for further research in other hospital institutions, as well as providing better and more appropriate care for the identified type.

\section{KEYWORDS}

Intensive Care Units; Critical Care; Morbidity and mortality indicators.

\section{RESUMEN}

El objetivo de este estudio es caracterizar el perfil clínico y sociodemográfico de los pacientes ingresados en una Unidad de Cuidados Intensivos de un hospital en el estado de Sergipe. Esta es una sección transversal de una cohorte prospectiva vinculada al proyecto titulada UTISE. La recopilación de datos se realizó diariamente, entre octubre / 2018 y mayo / 2019, y se realizó a través del análisis de registros médicos electrónicos. La muestra de investigación fue por conveniencia y correspondió a pacientes ingresados durante el período de recolección de datos. Se incluyeron 43 pacientes, predominantemente hombres (55.8\%), con una edad promedio de $68 \pm 19,25$ años, peso de $67.53 \pm 13,28 \mathrm{~kg}$, índice de masa corporal de $24,66 \pm 6,38 \mathrm{~kg} / \mathrm{m}^{2}$, altura entre $1.60-1,69 \mathrm{~m}$, la mayoría de los pacientes que vienen de la sala de emergencias $66.1 \%$, con trastornos cardiovasculares $25.1 \%$ siendo la razón principal de hospitalización. A partir de la identificación de este perfil, es posible utilizar los resultados de este estudio como un subsidio para futuras investigaciones en otras instituciones hospitalarias, así como brindar una atención mejor y más adecuada para el tipo identificado.

\section{PALABRAS CLAVE}

Unidades de cuidados intensivos; Cuidado crítico; Indicadores de morbilidad y mortalidad. 


\section{INTRODUÇ̧̃̃o}

A Unidade de Terapia Intensiva (UTI) consiste em um setor hospitalar que envolve uma equipe multiprofissional à uma densidade tecnológica de alta complexidade, o que permite a promoção de uma assistência qualificada, integralizada, holística e eficaz ao paciente crítico. Recomenda-se que 7 a 15\% do total de leitos do hospital sejam destinados para UTI, levando-se em consideração as características próprias de cada instituição (MELO et al., 2014).

As características prevalentes do perfil clínico e sociodemográfico identificado em estudos publicados no Brasil são de pacientes críticos, do sexo masculino, com faixa etária acima de 60 anos, tendo como procedência as Unidades de Pronto Atendimento seguida do Centro-Cirúrgico, com presença de morbidades associadas e um tempo de internação de aproximadamente de 10 dias (ACUÑA, 2007; MOLINA, 2008; RODRIGUEZ, 2016).

Assim, o conhecimento sobre o perfil da clientela assistida em UTI é importante, pois facilita o planejamento do processo de assistência à saúde dos pacientes, direciona o cuidado para esse tipo de cliente, como também melhora a utilização dos recursos disponíveis (FREITAS, 2010). A assistência promovida reflete em benefícios para o processo do cuidar mais humanizado, melhor organização do serviço e, consequentemente, redução de gastos por meio da implementação custo-efetividade (MELO et al., 2014).

A partir do conhecimento do perfil clínico e sociodemográfico dos pacientes assistidos na UTI, o planejamento de ações pode ser realizado e estruturado, visando a melhoria da assistência, a racionalização no uso de recursos materiais e consequentemente a diminuição do número de internações (PRECE et al., 2017). Assim, foram levantados dados locais da assistência prestada a pacientes críticos, com este intuito. Deste modo, definiu-se como pergunta de pesquisa: Como se configura o perfil dos pacientes atendidos em UTI? Sendo o objetivo deste estudo caracterizar o perfil clínico e sociodemográfico de pacientes admitidos em uma UTI de um hospital privado do estado de Sergipe.

\section{MÉTODO}

Trata-se de um corte transversal de uma coorte prospectiva vinculada ao projeto intitulado UTISE, realizado em um hospital privado localizado em Aracaju/ SE. O referido hospital possui UTI clínica e cirúrgica, a primeira é dividida em 10 leitos para casos agudos e 10 para casos crônicos e a segunda possui 10 leitos, mas apenas 6 leitos estavam ativos durante o período da coleta de dados.

A amostra da pesquisa foi por conveniência e correspondeu aos pacientes que foram admitidos na UTI durante o período de coleta de dados. Os critérios de inclusão consistiram em pacientes admitidos na UTI durante o período de coleta de dados, com idade maior ou igual a 18 anos e tempo de permanência mínima de 24 horas. Os critérios de exclusão são pacientes que evoluíram para alta/ transferência da UTI para outra unidade ou óbito nas primeiras 24 horas do internamento. 
A coleta de dados ocorreu diariamente, entre outubro de 2018 e maio de 2019, sendo realizada por meio da análise dos prontuários eletrônicos, por acadêmicos de enfermagem e enfermeiros, capacitados previamente.

Para a caracterização sociodemográfica foram utilizadas algumas variáveis do instrumento de coleta de dados próprio do projeto UTISE, como idade, sexo, peso, altura e Índice de Massa Corpórea (IMC) e a caracterização clínica foram observadas a presença de comorbidades segundo a Classificação Internacional de Doenças (CID-10) o setor hospitalar de origem, tempo de internação, suporte admissional (medicações e dispositivos em uso) e mortalidade na UTI.

Os dados obtidos foram inseridos em tabelas no programa Microsoft Excel 2010, exportados e submetidos à análise estatística no programa Statistical Package for the Social Sciences (SPSS) versão 21.0. As análises incluíram estatística descritiva para a apresentação das características clínicas e sociodemográficas da amostra e coeficiente de correlação de Pearson para análise de correlação entre tempo de internação e óbito, tendo como nível de significância p<0,05.

A pesquisa teve início após aprovação do projeto pelo Comitê de Ética e Pesquisa, parecer número 2.830.187, com a anuência da instituição participante e após assinatura do Termo de Consentimento Livre e Esclarecido (TCLE) pelo paciente, familiar ou outro responsável legal.

\section{RESULTADOS}

A análise do trabalho foi realizada a partir de uma amostra de 43 pacientes. Os resultados foram organizados em duas categorias: perfil sociodemográfico e perfil clínico. 0 perfil sociodemográfico foi identificado a partir de cinco variáveis: idade, sexo, peso, altura e IMC (Tabela 1).

A faixa etária mais encontrada está entre os anos de 80 a 89 anos com 23,4\% $(n=10)$, seguida por 40 e 49 anos e 60 e 69 anos, ambos com 18,6\% (n=8).

Tabela 1 - Dados sociodemográficos dos pacientes admitidos na UTI de um hospital privado entre outubro de 2018 e maio de 2019, Aracaju, SE

\section{Variável}

\begin{tabular}{cc}
\hline Sexo & $\mathrm{n}(\%)$ \\
Masculino & $24(55,8 \%)$ \\
Feminino & $19(44,2 \%)$ \\
\hline
\end{tabular}

média \pm desvio padrão

Idade $\quad 68,0 \pm 19,3$

Peso $\quad 67,5 \pm 13,3$ 


\begin{tabular}{cc}
\hline Variável & \\
Altura & $\mathrm{n}(\%)$ \\
1,40 a $1,49, \mathrm{n}(\%)$ & $3(7,0 \%)$ \\
1,50 a $1,59, \mathrm{n}(\%)$ & $11(25,6 \%)$ \\
1,60 a $1,69, \mathrm{n}(\%)$ & $17(39,5 \%)$ \\
1,70 a $1,79, \mathrm{n}(\%)$ & $11(25,6 \%)$ \\
$>1,80, \mathrm{n}(\%)$ & $1(2,3 \%)$ \\
\hline & média \pm desvio padrão \\
\hline IMC & $24,7 \pm 6,4$ \\
\hline
\end{tabular}

Fonte: Dados da Pesquisa (2019).

Conforme apresentado na Figura 1, os pacientes foram provenientes da emergência $(65,1 \%)$ devido a distúrbios cardiovasculares (25,6\%) em sua maioria. A Tabela 2 traz as informações quanto à admissão na UTI.

Figura 1 - Distribuição espacial do número de pacientes admitidos na UTI de um hospital privado, segundo procedência da internação, entre outubro de 2018 e maio de 2019, Aracaju, SE

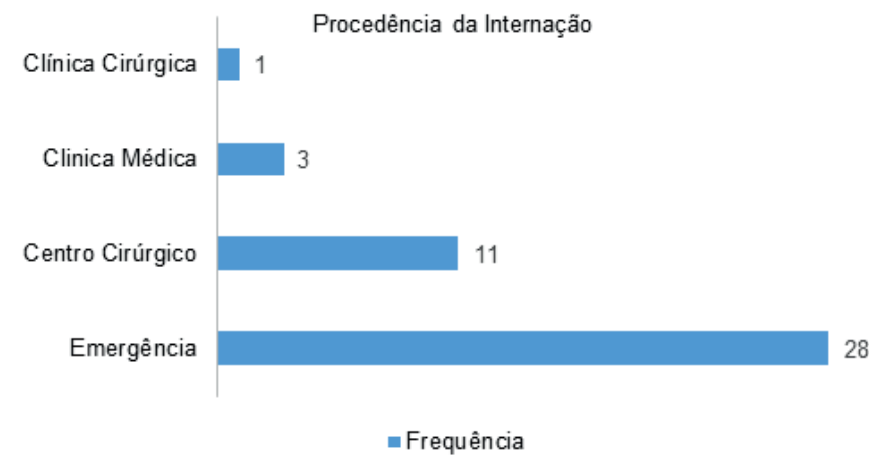

Fonte: Dados da Pesquisa (2019).

Tabela 2 - Diagnóstico admissional de pacientes admitidos na UTI de um hospital privado, entre outubro de 2018 e maio de 2019, Aracaju, SE

\begin{tabular}{ccc}
\hline Variável & Frequência & Percentual (\%) \\
\hline Cardiovascular & 11 & 25,6 \\
Respiratório & 7 & 16,3 \\
\hline
\end{tabular}




\begin{tabular}{ccc}
\hline Variável & Frequência & Percentual (\%) \\
\hline Neurológico & 6 & 14,0 \\
Infeccioso & 6 & 14,0 \\
Digestório & 5 & 11,6 \\
Trauma & 4 & 9,2 \\
Endócrino-Metabólico & 2 & 4,7 \\
Renal & 1 & 2,3 \\
Neoplasia & 1 & 2,3 \\
Total & 43 & 100 \\
\hline
\end{tabular}

Fonte: Dados da Pesquisa (2019).

Quanto à presença de comorbidades, a mais prevalente foi Hipertensão Arterial Sistêmica (HAS) com 62,8\%, seguida por Dislipidemia (34,9\%), Arritmia (20,9\%) e Diabetes Mellitus (DM) (20,9\%), conforme apresentado na Tabela 3.

Tabela 3 - Frequência de morbidades de pacientes admitidos na UTI de um hospital privado, entre outubro de 2018 e maio de 2019, Aracaju, SE

\begin{tabular}{cc}
\hline Variável & $\mathbf{n}(\mathbf{1 0 0 \% )}$ \\
\hline Hipertensão Arterial Sistêmica (HAS) & $27(62,8 \%)$ \\
Dislipidemia & $15(34,9 \%)$ \\
Arritmia & $9(20,9 \%)$ \\
Diabetes Mellitus (DM) & $9(20,9 \%)$ \\
Infarto Agudo do Miocárdio (IAM) prévio & $6(14 \%)$ \\
Hipotireoidismo & $6(14 \%)$ \\
Acidente Vascular Encefálico prévio & $4(9,3 \%)$ \\
Doença Pulmonar Obstrutiva Crônica (DPOC) & $3(7 \%)$ \\
Doença Vascular Periférica (DVP) & $2(4,7 \%)$ \\
Doença Hepática & $1(2,3 \%)$ \\
\hline
\end{tabular}

Fonte: Dados da Pesquisa (2019).

O suporte admissional refere-se ao uso de drogas intravenosas e aos dispositivos em uso no paciente no momento ou até uma hora da admissão na UTI. Em relação a este suporte, o medicamento mais utilizado foi fentanil e o dispositivo foi a sonda vesical de demora (SVD) (Tabela 4). 
Tabela 4 - Suporte admissional de pacientes admitidos na UTI de um hospital privado, entre outubro de 2018 e maio de 2019, Aracaju, SE

\begin{tabular}{cc}
\hline Variáveis & $\mathbf{n}(\%)$ \\
\hline Drogas Vasoativas & \\
Dobutamina & $0(0 \%)$ \\
Noradrenalina & $5(11,6 \%)$ \\
Drogas Sedativas & \\
Midazolam & \\
Fentanil & $4(9,3 \%)$ \\
Dispositivos em uso & $6(14 \%)$ \\
Sonda Vesical de Demora & \\
Cateter Venoso Central & \\
Sonda Nasoenteral & $17(39,5 \%)$ \\
Tubo Orotraqueal & $12(27,9 \%)$ \\
Sonda Nasogástrica & $9(20,9 \%)$ \\
Dreno & $7(16,3 \%)$ \\
Traqueostomia & $5(11,6 \%)$ \\
& $3(7,0 \%)$ \\
\hline
\end{tabular}

Fonte: Dados da pesquisa (2019).

0 tempo médio de internação destes pacientes na UTI foi $10 \pm 8$ dias. A partir desse dado foi possível estabelecer uma correlação positiva entre o tempo de internação e o óbito como o desfecho $(r=0,437, p=0,003)$.

\section{DISCUSSÃO}

O aumento da demanda por serviços de atendimento especializado fez com que aumentasse o número de UTI por todo o mundo, sendo necessário identificar o perfil de pacientes que mais necessitam do cuidado intensivo (MOLINA et al., 2008). Partindo deste ponto, foi traçado o perfil sociodemográfico e clínico de pacientes admitidos em uma UTI particular de Sergipe. Evidenciou-se predominância do sexo masculino, achado também observado por estudos realizados no estado de Santa Catarina (61,6\%) (PRECE et al., 2017) e Paraná (55,8\%) (RODRIGUEZ et al., 2016).

Alguns autores apontam diversificados fatores para que homens tenham um maior predomínio em internações na UTI, dentre eles destacam-se os hábitos comportamentais e sociais, como etilismo, 
tabagismo, sedentarismo e a pouca procura aos serviços de promoção e prevenção a saúde (CAMUCI et al., 2014; ARAÚJO, 2013; GUIA et al., 2015).

Quanto à faixa etária, há uma prevalência de atendimento a pacientes mais idosos, com média de 68 anos e maior número de pacientes na faixa etária de 80 e 89 anos. 0 envelhecimento populacional faz com que se aumente a frequência de pacientes idosos com agravos a saúde, necessitando do tratamento em UTI (FAVARIN; CAMPONOGARA, 2012). Contudo, é válido ressaltar que este dado pode sofrer impacto da expectativa de vida da população onde o estudo foi realizado, como visto por Acuña e colaboradores (2007), no qual a faixa etária encontrada foi de 53 anos.

Quanto às medidas antropométricas identificadas neste estudo, não foi estabelecida classificação dos pacientes em magreza, atrofia e excesso de peso. Entretanto, percebe-se que pacientes internados em UTI tendem a ter condição nutricional reestabelecida, mas com peso corpóreo alterado. Esta condição tende a ser modificada para maior peso em virtude de edema consequente à resposta hemodinâmica aos distúrbios sistêmicos presentes, como também diminuída frente à restrição motora e perda de massa corpórea (FONTOURA et al., 2006).

Observou-se que a maioria dos pacientes avaliados procedeu da emergência (55,1\%). Araújo (2013) e Guia e colaboradores (2015) também constataram este dado, apresentando o setor de emergência como porta de entrada de pacientes que necessitam de cuidados intensivos, uma vez que este setor no ambiente hospitalar fornece atendimento aos pacientes com casos agudizados.

As principais causas de internação foram por distúrbios cardiovasculares e respiratórios, com a HAS como comorbidade mais presente. Dado este constatado por outros estudos, que também evidenciaram a predominância destas causas de internações (PRECE et al., 2017; GUIA et al., 2015). Com agravamento das doenças crônicas tais como HAS e DM, observado nos dias atuais, culmina a ocorrência destes problemas de saúde em maior número, impactando no tempo de internação e na taxa de mortalidade (GUIA et al., 2015; FAVARIN; CAMPONOGARA, 2012).

O tempo médio de internação hospitalar observado foi $10 \pm 8$ dias, contudo não foi feita correlação com faixa etária. Pois sabe-se que o tempo de internação hospitalar também está relacionado à faixa etária do paciente. Os resultados evidenciaram que pacientes com idade mais avançada tenham em torno de 13,7 dias de internação, enquanto os pacientes mais jovens tenham de cerca de 10 dias (ARAÚJO, 2013).

Como medida de redução do tempo de internação e taxa de mortalidade, o suporte terapêutico deve estar bem estabelecido e conduzido de forma precoce. Neste estudo, a análise foi feita no momento da admissão até a primeira hora de internação na UTI, sendo observado suporte farmacológico predominantemente com droga vasoativa, sedativo e analgésico. Tais medicamentos atuam, em sua maior parte de forma sistêmica objetivando a estabilidade hemodinâmica dos pacientes, ou aliviando a dor proporcionando o conforto (CAMUCI et al., 2014).

Ao analisar presença de dispositivos, foi observado mais frequente o uso de SVD e CVC. Estes são utilizados em maior frequência pela necessidade de controle hídrico rigoroso e necessidade de acesso calibroso de preferência instalado em vasculatura central que permita a administração de drogas vasoativas, respectivamente. 0 uso de dispositivos invasivos rompe a integridade cutaneomucosa, causando desequilíbrio em toda a microbiota ou alterações das estruturas orgânicas (ANGHINONI et 
al., 2018). Deste modo, faz-se necessário o uso de medidas protetivas para evitar maiores taxas de infecções e menor tempo de internação.

Em análise de correlação entre mortalidade e tempo de internação foi observada correlação positiva entre as duas variáveis, sendo evidenciado impacto do tempo de permanência na UTI com o desfecho de óbito. A permanência na UTI depende de alguns fatores como, compensação da doença de base, potencial de reversibilidade do quadro clínico e dependência a dispositivos invasivos (IOT, TQT) (OLIVEIRA et al., 2010). Recomenda-se que o tempo de internação em UTI seja curto, pois o tempo prolongado leva à ocorrência de eventos adversos, como a lesão por pressão e as infecções respiratórias relacionadas a assistência à saúde (WILLIAMS et al., 2010).

\section{CONSIDERAÇÕES FINAIS}

O estudo identificou predominância de homens, idosos, admitidos na UTI provenientes da unidade de emergência com acometimento cardiovascular. Ainda, foi observada correlação positiva entre tempo de internação e mortalidade. Deste modo, espera-se que o estudo sirva de base para novos estudos na área bem como fundamentação para melhoria da assistência aos pacientes críticos, tendo foco em uso de instrumentos e escores prognósticos que permitam a avaliação e conduta clínica precoce.

O estudo tem como limitação o tamanho da amostra, que ao se apresentar em número reduzido, permite considerar os resultados encontrados apenas para a população em questão.

\section{REFERÊNCIAS}

ACUÑA, K. et al. Características clínico-epidemiológicas de adultos e idosos atendidos em Unidade de Terapia Intensiva pública da Amazônia (Rio Branco, Acre). Rev. Bras. Terap. Intens. v. 19, n. 3, p. 304-309, 2007.

ANGHINONI, T. H. et al. Adesão ao protocolo de prevenção de infecção do trato urinário. Rev. Enferm. UFPE, v. 12, n. 10, p. 2675-2682, 2018.

ARAUJO, T. G. de et al. Readmissões e óbitos após a alta da UTI: um desafio da terapia intensiva.

Rev. Bras. Terap. Intens. v. 25, n. 1, p. 32-38, 2013.

CAMUCI, M. B. et al. Caracterização epidemiológica de pacientes adultos internados em uma unidade de terapia intensiva de queimados. Cogitare Enferm. v. 19, n. 1, 2014.

FAVARIN, S. S.; CAMPONOGARA, S. Perfil dos pacientes internados na unidade de terapia intensiva adulto de um hospital universitário. Rev. Enferm. UFSM, v. 2, n. 2, p. 320-329, 2012. 
FONTOURA, C. S. M. et al. Avaliação nutricional de paciente crítico. Rev. Bras. Terap. Intens. v. 18, n. 3, p. 298-306, 2006.

FREITAS, E. R. F. S. Perfil e gravidade dos pacientes das unidades de terapia intensiva: aplicação prospectiva do escore APACHE II. Rev. Latino-Am. Enferm. v. 18, n. 3, p. 317-323, 2010.

GUIA, C. M. et al. Perfil epidemiológico e preditores de mortalidade de uma unidade de terapia intensiva geral de hospital público do Distrito Federal. Com. Ciên. Saúde, v. 26, n. 01/02, p. 9-19, 2015.

MELO, A. C. L. et al. Perfil de pacientes de terapia intensiva: subsídios para a equipe de enfermagem. Rev Enferm. UFPE, v. 8, n. 9, p. 3142-3148, 2014.

MOLINA, R. C. M. et al. Caracterização das internações em uma unidade de terapia intensiva pediátrica, de um hospital-escola da região sul do Brasil. Ciên. Cuid. Saúde, v. 7, p. 112-120, 2008.

OLIVEIRA, A. B. F. de et al. Fatores associados à maior mortalidade e tempo de internação prolongado em uma unidade de terapia intensiva de adultos. Rev. Bras. Terap. Intens. v. 22, n. 3, p. 250-256, 2010.

PRECE, A. et al. Perfil de pacientes em terapia intensiva: necessidade do conhecimento para organização do cuidado. Cad. Esc. Saúde, v. 2, n. 16, p. 35-48, 2016.

RODRIGUEZ, A. H. et al. Características epidemiológicas e causas de óbitos em pacientes internados em terapia intensiva. Rev. Bras. Enferm. v. 69, n. 2, p. 229-234, 2016.

WILLIAMS, T. A. et al. Effect of length of stay in intensive care unit on hospital and long-term mortality of critically ill adult patients. Brit. J. Anaesth. v. 104, n. 4, p. 459-464, 2010. 
1 Enfermeiro, Universidade Tiradentes - UNIT.

E-mail: clei.ribeiro14@gmail.com

2 Enfermeira, Universidade Tirade- UFS;ntes - UNIT.

E-mail: marcos_bioenf@hotmail.com

3 Mestre em Enfermagem pela Universidade Federal de Sergipe - UFS; Enfermeira.

E-mail: krislainy_passos@hotmail.com

4 Mestre em Ciências Aplicadas à Saúde pela Universidade Federal de Sergipe - UFS; Enfermeiro.

E-mail: shirleydosea@yahoo.com.br

5 Mestre em Ciências da Saúde pela Universidade Federal de Sergipe - UFS; Enfermeiro.

E-mail: lucas-saa@hotmail.com

6 Doutor em Ciências. Professor Adjunto, Departamento de Enfermagem, Campus Lagarto Universidade Federal de Sergipe - UFS; Enfermeiro.

E-mail: eduesley.santos@gmail.com

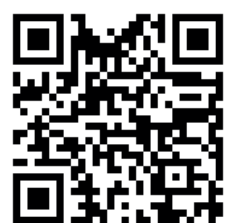

A autenticidade desse artigo pode ser conferida no site https://periodicos. set.edu.br

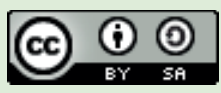

Este artigo é licenciado na modalidade acesso aberto sob a Atribuição-Compartilha Igual CC BY-SA

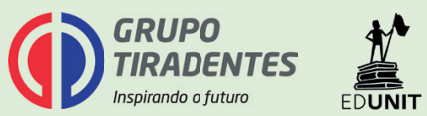

